African Crop Science Journal by African Crop Science Society is licensed under a Creative Commons Attribution 3.0 Uganda License. Based on a work at www.ajol.info/ and www.bioline.org.br/cs DOI: https://dx.doi.org/10.4314/acsj.v29i3.2

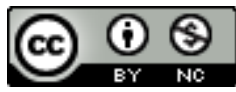

\title{
EFFICACITÉ DES TECHNIQUES DE GREFFAGE POUR L'ADÉQUATION AUX PORTE-GREFFES DE CAJOU
}

\author{
D. YÉLOUASSI, E. AKPO, A. ADANDONON et I. BALOGOUN
}

Ecole de Gestion et de Production Végétale et Semencière, Université Nationale d'Agriculture, BP: 43 Kétou, Bénin

Auteur correspondant : yeldaniustol@gmail.com

(Received 21 May 2021; accepted 19 August 2021)

\section{RESUME}

En absence de semences graines performantes capables d'assurer l'installation de plantations hautement productives, la multiplication végétative des plants d'anacardier reste une alternative viable pour obtenir des plantations homogènes et productives au Bénin. Pour ce fait, deux méthodes de greffage sont davantage utilisées par les pépiniéristes pour la production de plants greffés à savoir : la méthode en fente terminale et la méthode par placage. Cette étude a été menée pour étudier l'influence de ces techniques et de l'origine du greffon sur la croissance et l'aptitude au greffage des porte-greffes en période sèche au Bénin. L'étude a été menée à l'Université Nationale d'Agriculture du Bénin d'août 2019 à février 2020. Les porte-greffes utilisés, ont été produits à partir de semences certifiées du Centre de Recherches Agricoles-Centre du Bénin. Les traitements comprenaient la technique de greffage avec deux variantes (greffage par placage et greffage en fente terminale), et l'origine du greffon avec trois variantes (greffon d'Adakplamè, greffon de Sodji et greffon d'Agozounmè). Les mesures de protection phytosanitaires ont été prises et ont consisté en un traitement de toutes les unités parcellaires avec du Topbio pour combattre les insectes défoliateurs qui ont attaqué les plants greffés au cours de l'essai. Les résultats ont révélé que l'origine du greffon a une influence significative $(\mathrm{P}<0.05)$ sur la survie et la croissance en hauteur des plants greffés mais n'a aucun effet significatif sur la reprise et la croissance en diamètre de ces plants. Par contre, la technique de greffage a un effet significatif $(\mathrm{P}<0.005)$ sur la reprise et la survie des plants greffés ainsi que sur la croissance en hauteur et en diamètre de ces plants. En effet, un taux de reprise de 64,18 et 52,92\% a été observé respectivement pour le greffage par placage et le greffage en fente terminale mais seulement 44,81 et $34,65 \%$ des plants ont survécu respectivement pour le greffage par placage et le greffage en fente terminale, un mois après le greffage. En ce qui concerne la croissance des plants greffés, un accroissement moyen de $7,01 \mathrm{~cm}$ en hauteur et $1,15 \mathrm{~mm}$ en diamètre pour le greffage par placage contre $9,18 \mathrm{~cm}$ en hauteur et $1,55 \mathrm{~mm}$ en diamètre pour le greffage en fente terminale a été observé. Ces résultats stipulent que le greffage par placage est plus indiqué en saison sèche pour avoir un bon taux de réussite tandis que le greffage en fente terminale est plus indiqué pour une croissance rapide en hauteur et en diamètre des plants greffés.

Mots Clés: Anacardier, fente terminale, greffage par placage, croissance, productivité 


\begin{abstract}
In the absence of high-performance seeds capable of ensuring the installation of highly productive plantations, vegetative propagation of cashew seedlings remains a viable alternative for obtaining homogeneous plantations with productivity in Benin. For this reason, two grafting methods namely; terminal slot and in grafted seedlings are more used by nursery operators for the production of grafted seedlings. The objective of this study was to investigate the influence of these techniques and the origin of the graft on growth and grafting ability of rootstocks in the dry period in Benin. The study was conducted at the National University Agriculture of Benin during August 2020 to February 2021. The rootstocks used were produced from certified seeds from the Agricultural Research Center in Benin. Treatments included the grafting technique with two variants (grafting by veneer and terminal slot grafting), and the origin of the graft with three variants (Adakplamè graft, Sodji graft and Agozounmè graft). Plants were sprayed with Topbio to control defoliating insects during the experiments. The results revealed that the origin of the graft had a significant influence $(\mathrm{P}<0,05)$ on the survival and height growth of grafted plants, but had no significant effect on the recovery and the diameter growth of these plants. On the other hand, the grafting technique had a significant effect $(\mathrm{P}<0,005)$ on the recovery and survival of the grafted plants, as well as on the height and diameter growth of these plants. A recovery rate of 64.18 and $52.92 \%$ was observed, respectively, for the grafting by veneer and the grafting in terminal slot. One month after grafting, only 44.81 and $34.65 \%$ of the plants survived, respectively for the grafting by veneer and terminal slot grafting. With regard to the growth of the grafted plants, an average increase of $7.01 \mathrm{~cm}$ in height and $1.15 \mathrm{~mm}$ in diameter for the veneer grafting against $9.18 \mathrm{~cm}$ in height and $1.55 \mathrm{~mm}$ in diameter for the terminal slit grafting was observed. These results stipulate that grafting by veneer is more appropriate in the dry season to have a good success rate; while grafting in terminal slot is better for rapid growth in height and diameter of the grafted plants.
\end{abstract}

Key Words: Cashew tree, grafted seedlings, growth, productivity, terminal slot

\section{INTRODUCTION}

L'anacardier (Anacardium occidentale L.) est un arbre fruitier d'origine tropicale, résistant aux fortes chaleurs, mais très sensible aux basses températures (Lacroix, 2003). Généralement trouvé à des altitudes assez basses, l'anacardier est un arbre très rustique (Lawal et al., 2010) qui a de faibles exigences pédologiques et climatiques (Sedia, 2001). En effet, l'anacardier s' adapte à une large gamme de sols incluant les sols sableux, latéritiques et rocheux, et pousse sous des conditions climatiques très variées. Autrefois utilisée pour le reboisement, l'anacardier est actuellement plus cultivé pour son fruit qu'est la noix d'anacarde (Lacroix, 2003). La production d'anacarde est une importante activité économique dans beaucoup de pays tropicaux (De Figueiredo et al., 2001 ; Das et al., 2004 ;
Santos et al., 2007). En effet, la noix d'anacarde qui est le principal produit commercial de l'anacardier (Martinez et al., 2011), est un produit de rente utilisé dans divers domaines dont l'agroalimentaire, la cosmétologie, la médecine, l'industrie automobile. (Aliyu, 2007). Aussi, contribue-til énormément à l'économie des pays en voie de développement. Au cours des 20 dernières années, l'Afrique de l'Ouest a connu une croissance considérable de la production de noix de cajou, avec des augmentations de près de $10 \%$ par an ; qui est devenue sa deuxième ressource agricole d'exportation à partir de 2012. En 2015, la sous-région était classée première zone de production dans le monde, avec plus de 1.350.000 tonnes métriques de noix brutes devant l'Asie (Inde, Vietnam, Cambodge, Indonésie), qui reste autour des 1.300.000 tonnes métriques (Eteka et Faaki, 
2017). Par ailleurs, le Bénin fait partie de ces pays de la sous-région où la filière anacarde est l'un des piliers de développement à haute valeur ajoutée à promouvoir, dans le cadre du Programme d'Actions du Gouvernement. Pour ce pays, l'anacardier représente actuellement la deuxième culture d'exportation après le cotonnier (Yabi et al., 2013 ; Balogoun et al., 2014). En effet, de 36.487 tonnes métriques de noix brutes en 2001, l'exportation a atteint 116.398 tonnes métriques en 2008 (PAC/ DCM/SESP, 2009), avec une superficie estimée à 190.000 ha (FAO, 2009), et environ 146.332 tonnes métriques en 2011 (ACA, 2012). En termes de contribution, l'anacarde représente $24,87 \%$ de la valeur totale des exportations, $7 \%$ du PIB agricole et $3 \%$ du PIB national (Tandjiékpon, 2010 ; Balogoun et al., 2014). En 2011, le Bénin occupait la sixième place au plan mondial avec environ $3,8 \%$ du volume de noix produites et le troisième rang parmi les pays ouest-africains après le Nigéria et la Côte d'Ivoire (FAO, 2014). Actuellement, le Bénin s'est déjà hissé à la deuxième place derrière la Côte d'ivoire et devant la Tanzanie (FAO, 2020). L'anacardier assure aux producteurs des revenus réguliers nécessaires à l'amélioration de leurs conditions de vie (Djaha et al., 2014). Mais malgré l'importance de la production d'anacarde au Bénin, les rendements en noix des vergers demeurent faibles, de l'ordre de 350 à $600 \mathrm{~kg}$ ha $^{-1}$ (Adégbola et al., 2005), à cause des plantations créées avec du matériel végétal non amélioré et des pratiques culturales paysannes inadaptées. Ces rendements sont très faibles comparativement aux $820 \mathrm{~kg} \mathrm{ha}^{-1}$ obtenus chaque année en Inde (Thimmappaiah et al., 2009 ; Dasmohapatra et al., 2014). Pour lever cette contrainte, il est envisagé de produire des plants greffés avec des clones hauts producteurs comme ce fut le cas en Inde, au Brésil, au Vietnam, au Mozambique et en Tanzanie, et de mettre ces plants à la disposition des producteurs. Le greffage est en effet une technique de multiplication végétative couramment utilisée pour combiner différents matériels pour l'amélioration de l'anacardier (Ananthakrishnan et al., 2001). Cette technique permet d'avoir des vergers hautement productifs et homogènes à partir de plants greffés. Dans l'optique d'augmenter la productivité des plantations paysannes du Bénin, l'Institut National des Recherches Agricoles du Bénin (INRAB) a, par le biais du Centre de Recherches Agricoles-Centre du Bénin, sélectionné des arbres élites d'anacardiers et formé des pépiniéristes sur les techniques de greffage afin de mettre à disposition des producteurs des plants greffés obtenus à partir de ces arbres élites pour l'installation de leurs plantations (N'Djolossè et al., 2015). La demande étant très élevée, le greffage est devenu au fil du temps une activité génératrice de revenus dans laquelle plusieurs personnes se sont engagées comme étant des «pépiniéristes privés ». Cependant, l'offre de plants greffés demeure malgré tout faible en raison de la non-maîtrise des conditions de réussite du greffage de l'anacardier par la plupart de ces pépiniéristes privés, mais aussi à cause du cycle de production des plants greffés qui est relativement long et ne permet pas souvent de diffuser les plants produits dans la même année en ces temps de changements climatiques. Ce qui engendre un coût d'entretien des stocks des plants en pépinière. La production de plants greffés en plein temps, et surtout en saison sèche (période défavorable pour le greffage) s'impose de plus en plus aux pépiniéristes.

L'objectif du présent travail est d'étudier l'aptitude à la combinaison de porte-greffes d'anacardier suivant différentes techniques de greffage et l'origine des greffons.

\section{MATERIELS ET METHODES}

Matériel végétal. Des semences graines certifiées provenant d'arbres élites (arbres d'origines Dani/Katakou) installés au Centre de Recherches Agricoles-Centre (CRA-Centre) de Savè, ont été utilisées pour produire des porte-greffes de qualité sur lesquels le greffage 
a été réalisé. Les greffons utilisés ont été prélevés sur trois différents arbre-élites (Adakplamé $\mathrm{n}^{\circ} 24$, Agozounmè $\mathrm{n}^{\circ} 6$ et Sodji $\mathrm{n}^{\circ} 7$ ) provenant de trois différentes localités de la commune de Kétou (Bénin). Ces arbres étaient installés dans des plantations paysannes, sélectionnés en 2011 par l'Institut National des Recherches Agricoles du Bénin (INRAB, 2013) grâce à leurs caractéristiques performantes.

Dispositif expérimental. Nous avions installé un dispositif expérimental de Fisher pour cette étude (Fig. 1). Il s'agit d'un dispositif en bloc aléatoire complet à deux facteurs avec six traitements et quatre répétitions. Bien que réaliser en milieu contrôlé, le dispositif de Fisher a été choisi pour réduire l'influence d'éventuels facteurs d'hétérogénéité du site d'expérimentation. Les deux facteurs étudiés pour tester le greffage de l'anacardier étaient la provenance du greffon avec trois modalités à savoir arbre-élite n²4 d'Adakplamé (Ad24), arbre-élite $\mathrm{n}^{\circ} 6 \mathrm{~d}$ 'Agozounmè (Ag6) et arbreélite $\mathrm{n}^{\circ} 7$ de Sodji (So7); le deuxième facteur est la technique de greffage avec deux modalités : greffage par fente terminale (GF) et greffage par placage sur le côté (GP).

Les six traitements évalués étaient :

(i) Greffage par Fente terminale des greffons provenant de l'arbre-élite $\mathrm{n}^{\circ} 24$ d'Adakplamé (GFAd24);

(ii) Greffage par Fente terminale des greffons provenant de l'arbre-élite $\mathrm{n}^{\circ} 6$ d'Agozounmè (GFAg6);

(iii) Greffage par Fente terminale des greffons provenant de l'arbre-élite $\mathrm{n}^{\circ} 7$ de Sodji (GFSo7);

(iv) Greffage par Placage des greffons provenant de l'arbre-élite $\mathrm{n}^{\circ} 24$ d'Adakplamé (GPAd24);

(v) Greffage par Placage des greffons provenant de l'arbre-élite $\mathrm{n}^{\circ} 6$ d'Agozounmè (GPAg6); et

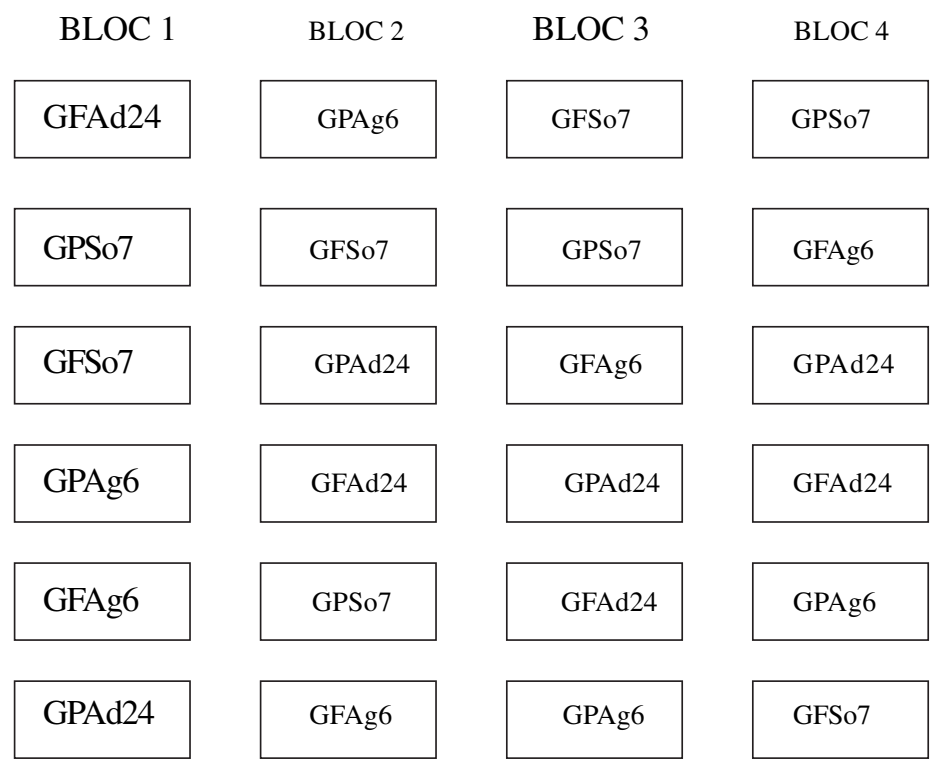

Figure 1. Schéma du dispositif expérimental. 
(vi) Greffage par Placage des greffons provenant de l'arbre-élite $n^{\circ} 7$ de Sodji (GPSo7)

Après la mise en place du dispositif, une ombrière a été construite pour créer un abri favorable aux plants (Fig. 2). L'ombrière a été clôturée par un grillage afin de protéger les plants contre les rongeurs et les vents forts. Les mesures de protection phytosanitaires ont été également prises et ont consisté en un traitement de toutes les unités parcellaires avec du Topbio, payé à Agro Solution-Bénin Sol pour combattre les insectes défoliateurs qui ont attaqué les plants greffés au cours de l'essai.

Production des porte-greffes. Avant le semis, la qualité des semences a été évaluée par la technique de flottaison avec de l'eau salée (renouvelée toutes les six heures pour éviter l'asphyxie). Seules les noix immergées (noix saines, grosses et bien remplies) ont été retenues pour la conduite de l'expérimentation. Les noix sont semées dans un substrat composé de terre franche, de sable et du fumier dans des pots en sachets biodégradables, perforés sur les côtés et à la base. Ces noix ont été enfouies dans le substrat préalablement mouillé, à $3 \mathrm{~cm}$ de profondeur, l'attache pédonculaire vers le haut et légèrement inclinée. Vingt pots ont été semés (une noix/pot) pour chacun des six (06) traitements. Ces traitements ont été répétés quatre fois soit au total 480 pots pour l'expérimentation ( $20 \times 4 \times 6=480$ pots).

Les pots ont été arrosés trois fois par jour pendant deux semaines puis la fréquence a été réduite à deux fois par jour. Les plants obtenus ont été traités deux fois à l'aide de TopBio pour prévenir les attaques des ravageurs. La levée de la totalité des graines a été constatée dans la troisième semaine après semis. Les plants sains obtenus ont servi de porte-greffes, une fois assez vigoureux (85 $5^{\text {èm }}$ jour après semis) pour la réalisation du greffage.

Réalisation du greffage. Des greffons de $10 \mathrm{~cm}$ ont été prélevés tôt le matin sur les arbres-élites d'anacardier d'Adakplamé (arbre $\mathrm{n}^{\circ} 24$ ), d'Agozounmè (arbre $\mathrm{n}^{\circ}$ ) et de Sodji (arbre $n^{\circ} 7$ ) et ont été conservés dans des serviettes mouillées pour préserver leur humidité. Ils ont été ensuite apposés sur les porte-greffes par les techniques de greffage en fente terminale et de greffage par placage sur le côté à l'aide des matériels de greffage appropriés. En effet, pour le greffage par fente terminale, une coupe horizontale franche a été préalablement faite sur les porte-greffes audessus du deuxième étage de feuilles (après 3 $\mathrm{cm}$ à partir du sommet) suivie d'une coupe longitudinale d'une profondeur de $3 \mathrm{~cm}$. Pour le greffage par placage, une fente oblique de 3 $\mathrm{cm}$ de profondeur a été faite sur le côté après avoir laissé $3 \mathrm{~cm}$ de hauteur. A partir du $7^{\text {ème }}$ jour après le greffage, l'égourmandage a été réalisé. Cette opération a consisté en l'élimination des feuilles appelées gourmandes qui poussent sur le sujet en dessous du lieu de fixation des greffons. Le 17ème jour après le greffage, les films plastiques, de couleur blanche, ayant servi à recouvrir les greffons dès leur pose sur les porte-greffes, ont été enlevés au fur et à mesure de l'apparition de

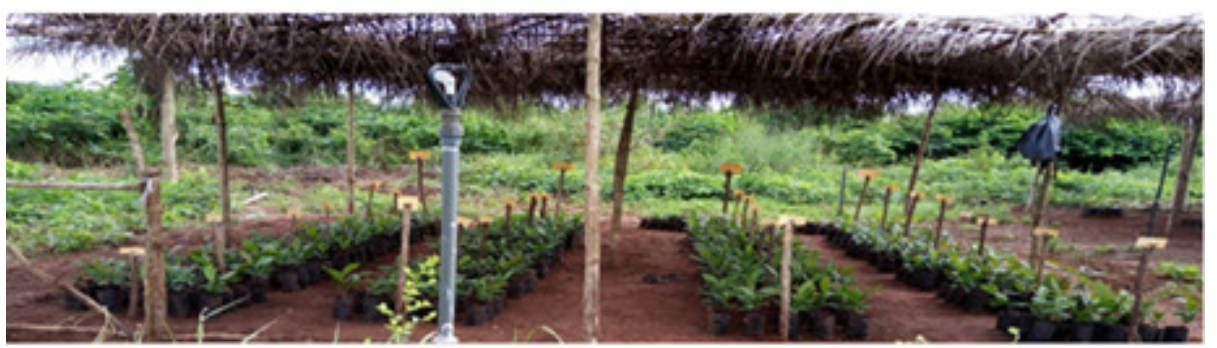

Figure 2. Vue de face du dispositif expérimental. 
jeunes feuilles sur les greffons. Les plants greffés obtenus sont arrosés deux fois par jour, en dehors des heures chaudes de la journée. La toute dernière reprise a été observée à la fin de la deuxième semaine.

Collecte et analyse des données. Des données ont été collectées sur tous les 480 plants à raison donc de 240 plants pour chaque technique de greffage (fente terminale et placage), 160 plants pour chaque origine de greffons (greffons des arbres Adakplamé n ${ }^{\circ} 24$, Agozounmè n ${ }^{\circ} 6$ et Sodji n ${ }^{\circ} 7$ ) et 80 plants pour chaque traitement (GFAd24, GFAg6, GFSo7, GPAd24, GPAg6, GPSo7). Deux types de données ont été collectées : (1) celles qui sont mesurées sur les porte-greffes avant le greffage à savoir la hauteur des porte-greffes, le diamètre au collet et le nombre de feuilles, et (2) les données qui sont collectées sur les plants greffés après greffage à savoir la hauteur des plants greffés, le diamètre au collet des plants greffés, le nombre de reprise, le nombre de plants ayant survécu, le nombre de bourgeon et le nombre de jeunes feuilles apparues. Le premier type (1) de données a été préalablement utilisé pour évaluer la vigueur et la croissance des porte-greffes afin d'estimer la date adéquate pour le greffage (date à laquelle tous les porte-greffes sont assez grands et vigoureux sans une différence significative). Ces données ont été collectées mensuellement après la levée totale. Le deuxième type (2) de données a été utilisé pour l'étude proprement dite, c'est-à-dire pour évaluer l'influence de l'origine du greffon et de la technique utilisée sur la réussite du greffage et la croissance des plants greffés. Ces données ont été collectées pendant un mois de façon hebdomadaire (tous les 7 jours) après le greffage. Après qu'une matrice de données soit créée dans le logiciel Excel, le nombre de reprise (après deux semaines) et le nombre de plants ayant survécu (après un mois) ont été respectivement utilisés pour calculer le taux de reprise et le taux de survie des plants greffés. Les statistiques descriptives telles que
: les valeurs moyennes, minimales, maximales, l'écart-type de la moyenne et le coefficient de variation ont été déterminées pour l'ensemble des variables quantitatives. Les données quantitatives enregistrées ont été soumises à l'analyse de variance (Anova) au seuil de $5 \%$ grâce au logiciel Statistica version 6 et la séparation des moyennes a été faite avec le test de DUNCAN à l'aide du logiciel $\mathrm{R}$ version 4.0.4. Ainsi, l'influence de la provenance des greffons, de la technique de greffage ainsi que leurs interactions ont été évaluées sur les paramètres tels que le taux de reprise (TR), le taux de survie (TS), le nombre de bourgeons (NB), le nombre de feuilles (NF), la hauteur moyenne (HMP) et le diamètre moyen des plants greffés (DMP).

\section{RESULTATS}

Influence de la provenance des greffons et des techniques de greffage sur les paramètres étudiés.

\section{Taux de reprise et de survie des plants} greffés. Les plants d'anacardier ont montré un taux de réussite de $58,54 \%$ soit 281 plants réussis sur 480 plants greffés (Tableau 1). Les résultats de l'analyse de variance montrent que la provenance des greffons n'a pas un effet significatif sur le taux de reprise des plants greffés ( $\mathrm{P}>0,05)$ (Tableau 2). Par contre, la reprise des plants greffés dépend de la technique de greffage utilisée $(\mathrm{P}<0,05)$ (Tableau 2). La technique de greffage par placage présente les meilleurs taux de reprise quel que soit l'origine du greffon (Fig. 3). Mais le taux de reprise le plus élevé $(80 \%)$ est obtenu lorsqu'on greffe les plants par placage en utilisant les greffons provenant d'Adakplamé. On constate aussi que les greffons provenant d'Adakplamé arbre ${ }^{\circ} 24$ (Ad24) présentent les meilleurs taux de reprise quel que soit la technique de greffage (Fig. 3).

Un mois après le greffage, le taux de survie total est d'environ $40 \%$ soit 193 plants survécus sur 480 plants greffés (Tableau 1). 
TABLEAU 1. Taux de reprise et taux de survie moyens par technique de greffage.

\begin{tabular}{lcc}
\hline & Taux de reprise $(\%)$ & Taux de survie $(\%)$ \\
\hline Fente terminale & $52,92 \mathrm{a}$ & $34,65 \mathrm{a}$ \\
Placage & $64,18 \mathrm{~b}$ & $44,81 \mathrm{~b}$ \\
Total & $58,54 \mathrm{ab}$ & $40,21 \mathrm{ab}$ \\
\hline
\end{tabular}

*Les moyennes de même colonne affectées de lettres différentes sont différentes à $95 \%$. Test DUNCAN, $\mathrm{R}$ version 4.0 .4

TABLEAU 2. Résultats des tests de significativité (t-test à $5 \%$ )

\begin{tabular}{llllll}
\hline Variables & \multicolumn{2}{c}{ Provenance } & & \multicolumn{2}{c}{ Technique de greffage } \\
\cline { 2 - 3 } & \multicolumn{1}{c}{$\mathrm{F}$} & $\mathrm{p}$ & & $\mathrm{F}$ & $\mathrm{p}$ \\
\hline Taux de reprise & 1,8886 & 0,163075 & & 5,009 & $0,008683^{*}$ \\
Taux de survie & 3,3962 & $0,042295^{*}$ & & 10,3058 & $0,002420^{* *}$ \\
Diamètre & 2,13 & 0,124360 & & 8,40 & $0,004669^{* *}$ \\
Hauteur & 3,176 & $0,046316^{*}$ & & 7,509 & $0,007347^{*}$ \\
Nombre de bourgeons & 0,281 & 0,756588 & & 0,007 & 0,932214 \\
Nombre de feuilles & 0,0561 & 0,945530 & & 0,0058 & 0,939655 \\
\hline
\end{tabular}

*Niveau de signification

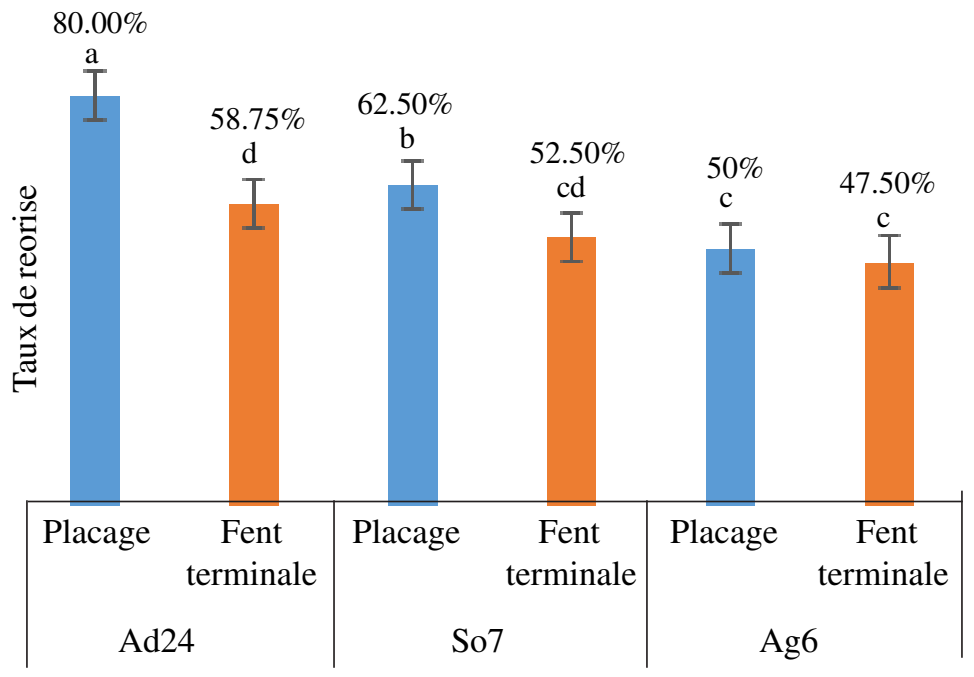

Provenance du greffon

Figure 3. Taux de réussite par type de matériel végétal. La provenance du greffon avec trois modalités à savoir arbre-élite n² 24 d'Adakplamé (Ad24), arbre-élite n 6 d'Agozounmè (Ag6) et arbre-élite $n^{\circ} 7$ de Sodji (So7). La barre représente l'erreur standard (SE). Test de DUNCAN à $5 \%$, R version 4.0.4. 
Les résultats du test de significativité ont montré que ce taux de survie des plants greffés dépend de la technique de greffage $(\mathrm{P}=0,002)$ et de l'origine des greffons utilisés $(P=0,042)$ (Tableau 2). En effet, la technique de greffage par placage a montré les meilleurs taux de survie par rapport au greffage en fente terminale quel que soit l'origine du greffon (Fig. 4). Les plants greffés avec les greffons d'Agozounmè (Ag6) ont présenté le taux de survie le plus élevé $(57,50 \%$ ) (Fig. 4).

Croissance des plants greffés. Les Figures 5 et 6, et le Tableau 3 présentent la croissance en hauteur et en diamètre des plants greffés un mois après greffage.
Croissance en hauteur des plants greffés. En un mois (de Semaine 0 à Semaine 4), la hauteur des plants greffés avec les greffons Ad24 est passée de 22,4 à $29,75 \mathrm{~cm}$, soit un accroissement de $7,35 \mathrm{~cm}$ pour la technique de placage et de 22,28 à $32,64 \mathrm{~cm}$, soit un accroissement de $10,36 \mathrm{~cm}$ pour la technique de fente terminale (Fig. 5). Celle des plants greffés avec les greffons $\mathrm{So} 7$ est passée de 22,31 à $29,26 \mathrm{~cm}$, soit un gain en hauteur de $6,95 \mathrm{~cm}$ pour la technique de placage et de 22,12 à $30,65 \mathrm{~cm}$, soit un accroissement de $8,53 \mathrm{~cm}$ pour la technique de fente terminale. Quant aux plants greffés avec les greffons Ag6, la hauteur a augmenté de $6,73 \mathrm{~cm}$ pour la technique de placage et $8,64 \mathrm{~cm}$ pour la

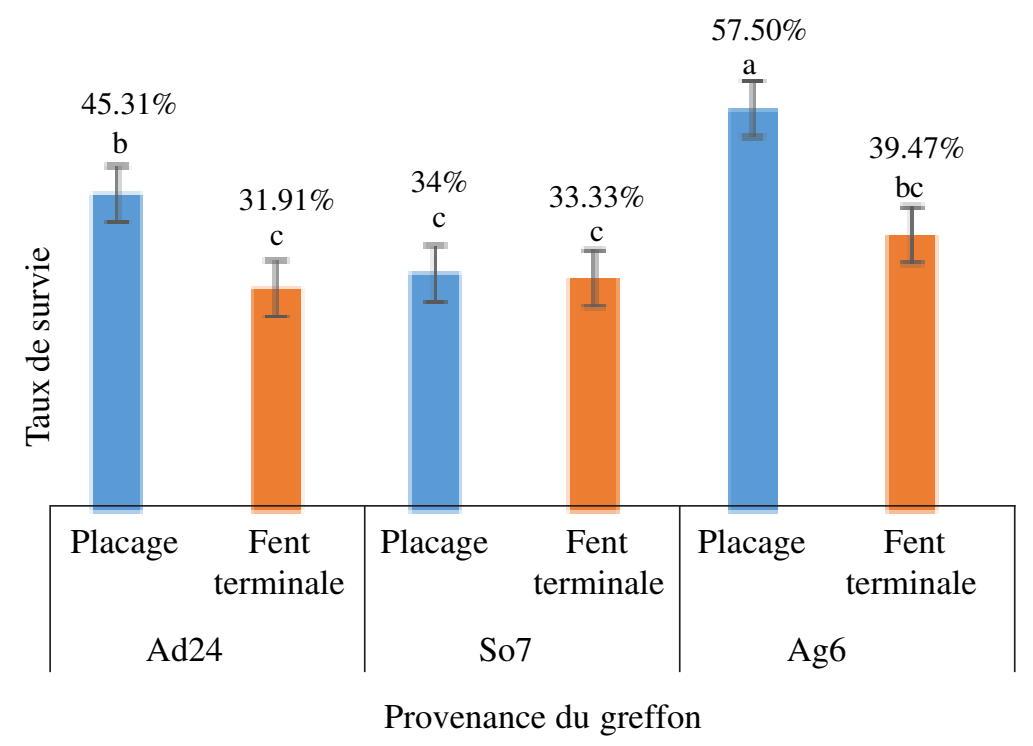

Figure 4. Taux de survie par type de matériel végétal. La provenance du greffon avec trois modalités à savoir arbre-élite n ${ }^{\circ} 4$ d'Adakplamé (Ad24), arbre-élite nº 6 d'Agozounmè (Ag6) et arbre-élite nº 7 de Sodji (So7). La barre représente l'erreur standard (SE). Test de DUNCAN à $5 \%$, R version 4.0.4.

TABLEAU 3. Croissance en hauteur et en diamètre des plants greffés en fonction du type de greffage

\begin{tabular}{lcc}
\hline Accroissement moyen & Hauteur $(\mathrm{cm})$ & Diamètre $(\mathrm{mm})$ \\
\hline Fente terminale & $9,18 \mathrm{a}$ & $1,55 \mathrm{a}$ \\
Placage & $7,01 \mathrm{~b}$ & $1,15 \mathrm{~b}$ \\
\hline
\end{tabular}

*Les moyennes de même colonne affectées de lettres différentes sont différentes à $95 \%$. Test DUNCAN, R version 4.0.4. 


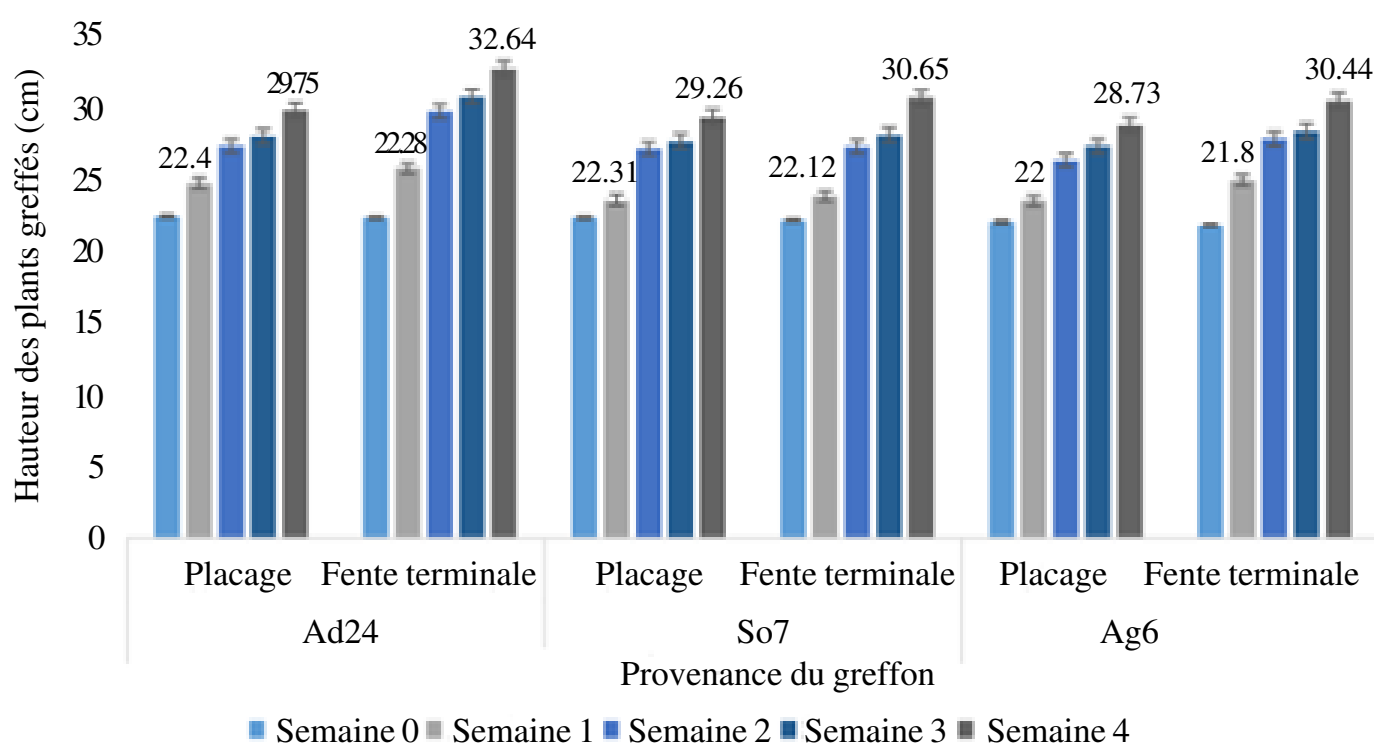

Figure 5. Variation de la croissance en hauteur des plants greffés.

La provenance du greffon avec trois modalités à savoir arbre-élite n²4 d'Adakplamé (Ad24), arbreélite $n^{\circ} 6$ d'Agozounmè (Ag6) et arbre-élite $n^{\circ} 7$ de Sodji (So7). La barre représente l'erreur standard (SE).

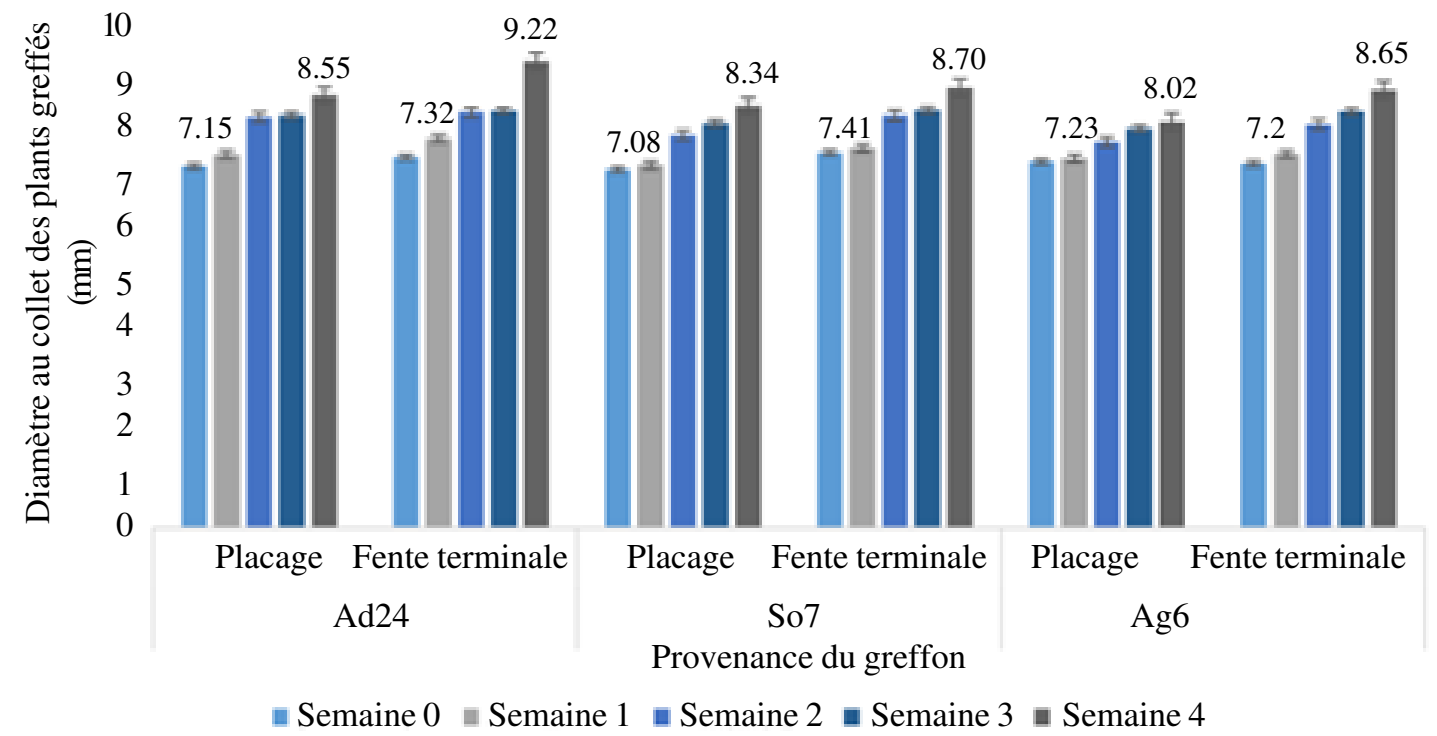

Figure 6. Variation du diamètre au collet des plants greffés.

La provenance du greffon avec trois modalités à savoir arbre-élite n²4 d'Adakplamé (Ad24), arbreélite $n^{\circ} 6$ d'Agozounmè (Ag6) et arbre-élite $n^{\circ} 7$ de Sodji (So7). La barre représente l'erreur standard (SE). 
technique de fente terminale. Les plants greffés avec des greffons provenant d'Adakplamé (Ad24) grandissent donc plus vite que ceux des deux autres types de greffons (Tableau 4). Cette différence observée entre la croissance des plants greffés avec les trois types de greffons est significative $(\mathrm{P}=0,046$ $<0,05$ ), au seuil de 5\% (Tableaux 2 et 4 ).

Par ailleurs, la technique de greffage influence fortement la croissance en hauteur des plants greffés. Une différence significative, au seuil de 5\%, a été observée au niveau de la hauteur des plants greffés $(P=0,007<0,05)$, selon chaque technique de greffage utilisée (Tableau 2). La technique de greffage par fente terminale favorise plus la croissance en hauteur des plants greffés que la technique de greffage par placage sur le côté.

Croissance en diamètre au collet des plants greffés. La croissance en diamètre au collet de la tige varie d'une technique de greffage à une autre. En effet, le diamètre au collet de la tige des plants greffés avec les greffons Ad24 qui était de 7,15 $\mathrm{mm}$ avant le greffage par placage est passé à $8,55 \mathrm{~mm}$ un mois après greffage, soit un accroissement de 1,4 $\mathrm{mm}$; alors qu'une augmentation de $1,9 \mathrm{~mm}$ a été observée au niveau de la technique de fente terminale (Fig. 6). Pour le greffage en fente terminale avec les greffons So7, le diamètre au collet des plants greffés est passé de 7,41 à
$8,70 \mathrm{~mm}$ au bout d'un mois (Semaine 0 à Semaine 4), soit un accroissement de 1,29 mm. Cet accroissement est légèrement supérieur au $1,26 \mathrm{~mm}$ observé pour le greffage par placage sur le côté. Il en est de même au niveau des greffons $\mathrm{Ag} 6$ où les plants ayant subi le greffage en fente terminale ont gagné $1,45 \mathrm{~mm}$ de plus en diamètre au collet un mois après le greffage tandis que ceux ayant subi le greffage par placage n'ont grossi que de $0,79 \mathrm{~mm}$ sur la même période. En comparant les trois types de greffons, on constate que ce sont les greffons Ad24 qui ont le meilleur gain en diamètre au collet $(1,9 \mathrm{~mm})$ après un mois quel que soit la technique de greffage utilisée. Les plants greffés avec des greffons provenant d'Adakplamé (Ad24) grossissent donc plus vite que les autres plants (Tableau 4). L'origine des greffons a un effet sur la croissance en diamètre au collet des plants.

Cependant, cette influence de la provenance des greffons sur le diamètre des plants greffés n'est pas significative au seuil de $5 \%(\mathrm{P}=0,12$ $>0,05)$ (Tableau 2). Néanmoins, une différence significative, au seuil de 5\%, a été observée entre le diamètre au collet des tiges des plants greffés par placage et ceux greffés en fente terminale $(P=0,004<0,05)$ (Tableau 2).

Appariation de bourgeons et de feuilles par semaine. Les plants greffés avec des greffons

TABLEAU 4. Hauteur et Diamètre moyens en fonction de l'origine des greffons et du type de greffage

\begin{tabular}{llcc}
\hline Technique de greffage & Origine & Hauteur $(\mathrm{cm})$ & Diamètre $(\mathrm{mm})$ \\
\hline \multirow{2}{*}{ Fente terminale } & Ad24 & $28,22 \mathrm{a}$ & 8,14 a \\
& So7 & 26,38 ab & 8,00 b \\
& Ag6 & 26,66 ab & 7,89 b \\
Placage & & & 7,87 b \\
& Ad24 & 26,42 ab & 7,67 c \\
& So7 & 25,97 ab & 7,61 c \\
\hline
\end{tabular}

*Les lettres qui sont les mêmes signifient qu'il n'y a pas de différence significative. Test de Duncan à $5 \%, \mathrm{R}$ version 4.0 .4 
provenant de Sodji (So7) produisent plus rapidement des bourgeons et de jeunes feuilles que les autres; soit 1,51 bourgeons et 2,47 feuilles par semaine (Fig. 7). Toutefois, l'apparition des bourgeons et des feuilles sur les plants après le greffage ne dépend ni de la technique de greffage ni de l'origine des greffons utilisés (Tableau 2).

\section{DISCUSSION}

Les résultats obtenus au cours de cette étude montrent que l'anacardier (Anacardium occidentale L.) s'adapte aux techniques de greffage telles que « le greffage par placage » et « le greffage en fente terminale » en saison sèche au Bénin. En effet, le greffage des plants d'anacardier dans sa période défavorable (en saison sèche) au Bénin a donné un taux moyen de survie d'environ $40 \%$ un mois après le greffage. Ce taux de survie obtenu est très faible comparativement aux $95 \%$ obtenus par Touré et al. (2017) au Sénégal en utilisant un matériel juvénile pendant la saison pluvieuse (période favorable au greffage). Cependant, nos résultats sont en accord avec ceux de Soloviev et al. (2004) qui ont aussi obtenu de faibles taux de succès au greffage sur le prunier d'Afrique (Sclerocarya birrea) en saison sèche. Le climat est donc un élément majeur qui influence la reprise et la survie des plants greffés. En effet, les travaux de greffage de la présente étude ont été effectués dans le mois de Décembre pendant la saison sèche. Ce mois durant lequel l'étude a été conduite est plus sec à cause de l'harmattan (Hala, 2011) avec pour corollaire une transpiration excessive des plants. Même si des arrosages ont été réalisés régulièrement, la perte d'eau par transpiration des plantes a probablement été plus grande que les apports d'eau, en raison de la faible humidité relative du moment (Djaha et al., 2012). Cette faible humidité relative qui caractérise le mois de Décembre provoque la déshydratation des greffons qui s'assèchent avant leur soudure aux porte-greffes. Bien que les jeunes sujets soient normalement très réactifs aux mécanismes qui conduisent à la soudure avec le greffon (Djaha et al., 2012), la période d'harmattan ne facilite pas cette soudure, encore que les greffons utilisés proviennent d'arbres adultes âgés de plus de 20 ans. Cela explique aussi le faible taux de survie des plants greffés car les greffons d'arbres adultes sont moins aptes au greffage à cause de leur vieillissement. Ces résultats

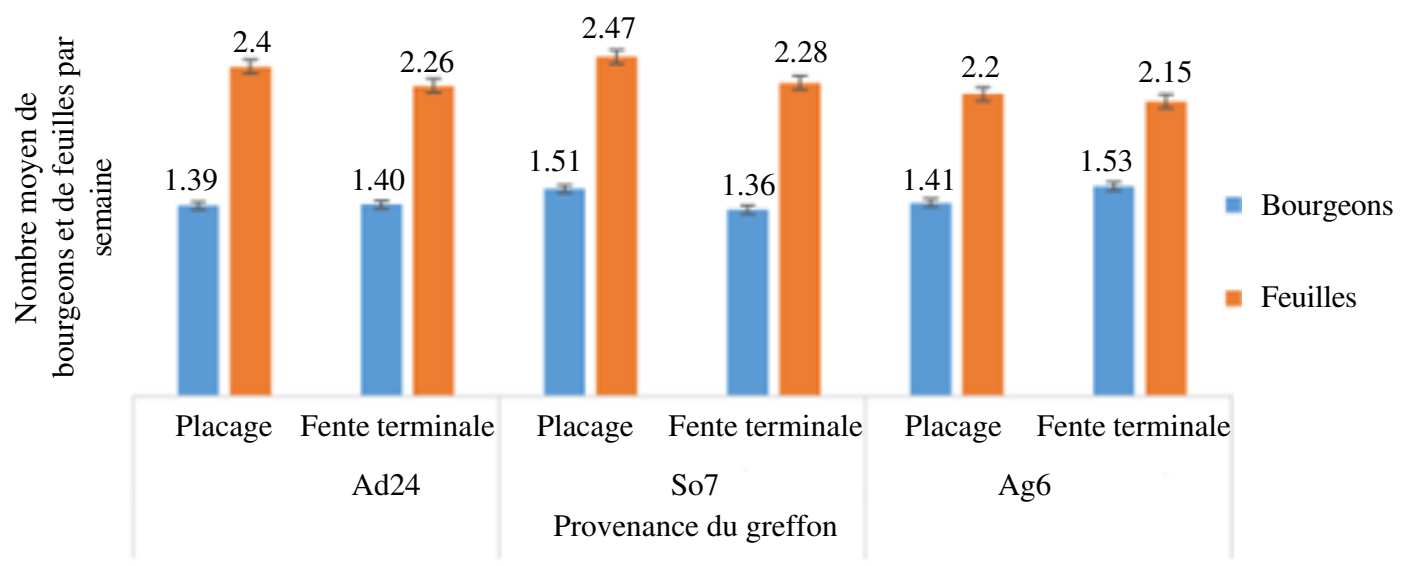

Figure 7. Fréquence absolue d'apparition des bourgeons et feuilles.

La provenance du greffon avec trois modalités à savoir arbre-élite $\mathrm{n}^{\circ} 24$ d'Adakplamé (Ad24), arbreélite ${ }^{\circ} 6$ d'Agozounmè (Ag6) et arbre-élite $n^{\circ} 7$ de Sodji (So7). La barre représente l'erreur standard (SE). 
corroborent ceux de Touré et al. (2017) qui avaient observé un taux de survie de $25 \%$ en saison pluvieuse lorsqu'ils utilisent des greffons provenant d'arbres adultes. En effet, le processus de vieillissement est un phénomène naturel qui affecte les végétaux ligneux au cours du temps, à travers les phases successives de leur développement ontogénétique à partir de la graine (Monteuuis, 1988). Une de ses conséquences majeures est la diminution, voire la perte de l'aptitude au clonage lorsque les arbres vieillissent. La connaissance et la maîtrise des différentes modalités de vieillissement sont donc un préalable aux études concernant la multiplication végétative des arbres adultes d'anacardiers. Par ailleurs, la technique de greffage par placage s'adapte mieux à ces diverses conditions par rapport à la technique de greffage en fente terminale. En effet, environ $45 \%$ des plants greffés par placage ont survécu contre environ $35 \%$ pour le greffage en fente terminale. Ces résultats corroborent ceux de Batamoussi et al. (2017), qui avaient obtenu au Nord-Bénin en saison sèche environ $52 \%$ et $25 \%$ de reprise respectivement pour la technique de greffage par placage et celle de greffage en fente terminale. Nos travaux montrent que la technique de greffage utilisée influence significativement $(P<0,05)$ la reprise et la survie des plants greffés, et que le greffage par placage réussit mieux en saison sèche comparativement au greffage en fente terminale. De telles observations ont été également faites par Bognina et al. (2019) au Burkina Faso. Dans leur essai, la méthode en fente terminale $(11,85 \%)$ s'était aussi révélée moins efficace que la méthode par placage de côté $(50,74 \%)$. Selon Bognina et al. (2019), le greffage en fente terminale réussit plus en saison pluvieuse, mais ne supporte pas les périodes sèches. Par contre, la saison n'a pas assez d'influence sur le greffage par placage de côté (Batamoussi et al., 2017). Le greffage par placage simple de côté permet de procurer aux greffons une humidité plus élevée comparativement au greffage en fente terminale. En effet, le greffage par placage simple de côté met en contact étroit le cambium du greffon avec le cambium du porte-greffe alors que pour le greffage en fente terminale, la surface de contact des cambiums des deux sujets est très réduite, ce qui justifie les résultats de Aliyu (2001) qui a obtenu un faible taux de succès du greffage en fente terminale entre 4 et $40 \%$.

Par ailleurs, les résultats de notre étude montrent que la provenance des greffons n'a pas un effet significatif sur la reprise des plants greffés et n'influence que faiblement la survie de ces plants. Cela suppose qu'il y a une compatibilité ou une bonne aptitude à la combinaison entre les différents matériels d'anacardiers du Bénin. Cependant, la technique de greffage utilisée a un effet significatif $(\mathrm{P}<0,05)$ sur la croissance en hauteur et en diamètre au collet des plants greffés. Un accroissement conséquent a été obtenu au niveau de la hauteur et du diamètre au collet des plants quel que soit le type de greffon lorsqu' on utilise la technique de greffage en fente terminale. En effet, la technique de greffage par fente terminale favorise plus la croissance en hauteur et en diamètre des plants greffés que la technique de greffage par placage sur le côté. Ceci s'explique par le fait que la sève produite par le plant greffé par placage, sert à nourrir le greffon d'une part mais aussi le support d'autre part jusqu'au 17 ème jour où le support sera amputé lors du démaillotage. Tandis que la sève produite au niveau des plants greffés par greffage en fente terminale est utilisée par la plante uniquement pour sa soudure et sa croissance. Ce qui justifie la croissance rapide en hauteur et en diamètre des plants lorsqu' on utilise la technique de greffage en fente terminale. Toutefois, selon Touré et al. (2017) cette technique de greffage en fente est moins efficace que la technique de greffage à l'anglaise simple qui, en termes de la croissance des plants greffés présente de meilleurs résultats. 
En ce qui concerne l'origine des greffons, les plants greffés avec des greffons provenant d'Adakplamé (Ad24) grandissent et grossissent plus vite que ceux des deux autres types de greffons. Ces greffons présentent également les meilleurs taux de reprise et de survie quel que soit la technique de greffage, comparativement aux autres. Cela peut s'expliquer par l'âge des sources de greffons ou par leur génotype. En effet, selon Bezerra (2007), le comportement de l'anacardier est significativement affecté par l'âge, le génotype et les conditions de culture, notamment le sol et le climat. Les greffons d'Adakplamè sont les plus compatibles aux porte-greffes de l'étude. L'origine génétique du greffon aurait donc une influence sur la croissance des plants greffés. Ces greffons ont probablement développé une plus grande affinité porte-greffe/ greffon ou aptitude à la combinaison comparativement aux autres types de greffons (De Larhoussile, 1980). Néanmoins, cette influence observée n'est pas significative pour le diamètre et est faiblement significative pour la hauteur des plants greffés.

\section{CONCLUSION ET PERSPECTIVES}

La filière anacarde joue un rôle très important dans l'économie béninoise. Elle constitue une source importante de revenus pour les divers acteurs et résout des problèmes socioéconomiques. Cependant, les rendements des plantations d'anacarde du Bénin sont encore très faibles comparativement aux rendements obtenus dans d'autres pays comme l'Inde. Il s'avère donc nécessaire d'hausser la productivité des plantations paysannes en utilisent de matériel végétal performant pour leurs installations. La production de plants d'anacardiers par voie végétative permet de multiplier fidèlement les caractéristiques des variétés ou morphotypes performants existants. Pour ce faire, deux techniques de greffage (placage de côté et fente terminale) sont souvent utilisées au Bénin. Le présent travail a permis d'étudier l'influence de ces techniques et de l'origine des greffons sur l'aptitude à la combinaison des différents matériels. Les résultats de cette étude ont révélé que la technique de greffage par placage est plus indiquée en saison sèche pour avoir un bon taux de réussite (reprise + survie) tandis que la technique de greffage en fente terminale est plus indiquée pour une croissance rapide en hauteur et en diamètre au collet des plants greffés. De ces résultats, il s'avère important d'expérimenter la technique de greffage à l'anglaise simple, qui est une technique qui pourrait réunir les avantages des deux techniques: greffage en fente terminale (absence de support) et greffage par placage (coupe oblique). Cela pourrait permettre d'avoir un bon taux de réussite et une croissance rapide en hauteur et en diamètre des plants greffés. Il est également important que des données soient prises sur les plants greffés lors de la phase de transplantation pour suivre l'influence de la technique de greffage et de l'origine du greffon sur la croissance des plants greffés durant la transition pépinièrechamp. La maîtrise de ce mécanisme permettra de mieux se positionner par rapport à la technique de greffage à utiliser pour avoir en temps record des plants greffés performants aptes à être transplantés pour l'installation de nouvelles plantations hautement productives.

\section{REMERCIEMENT}

Cette étude a été financée par l'Ecole de Gestion et de Production Végétale et Semencière (EGPVS) de l'Université Nationale d'Agriculture (UNA) que nous remercions sincèrement.

\section{REFERENCES BIBLIOGRAPHIQUES}

ACA, 2012. Annual Report 2011. African Cashew Alliance. 16pp.

Adégbola, P.Y., Oloukoï, L. et Sossou, H.C. 2005. Analyse de la compétitivité de la filière anacarde au Bénin. Rapport technique final. PAPA/INRAB, Bénin. 35pp.

Aliyu, O.M. 2001. Development of rapid method of propagating improved cultivars 
of cashew through budding, grafting and marcotting. Annual Research Report of the Cocoa Research Institute of Nigeria, Ibadan, Nigeria. pp. 30-31.

Aliyu, O.M. 2007. Clonal propagation in cashew (Anacardium occidentale L.): Effect of rooting media on the root-ability and sprouting of air-layers. Tropical Science 47:65-72.

Ananthakrishnan, G., Ravikumar, R., Girija, S. et Ganapathi, A. 2001. In vitro adventitious shoot formation from cotyledon explant of cashew (Anacardium occidentale L.). Scientia Horticulturae 93:343-355.

Batamoussi, M.H., Tokore Orou Mere, S.B. J., Moussa, I., Karami, O.M., Amanoudo, M-J. et Lawson, R.G. 2017. Contribution à l'amélioration du taux de réussite du greffage de l'anacardier (Anacardium occidentale) en pépinière dans la commune de Parakou au Nord-Bénin. International Journal of Biological and Chemical Sciences 11(5):2270-2276.

Balogoun, I., Saïdou, A., Ahoton, E.L., Amadji, L.G., Ahohuendo, C.B., Adebo, I.B., Babatoundé, S., Chougourou, C.D., Adoukonou-Sagbadja, H. et Ahanchede, A. 2014. Caractérisation des systèmes de production à base d'anacardier dans les principales zones de culture au Bénin. Agronomie Africaine 26(1):9-22.

Bello, S., Maliki, R., Adandonon, A., Yélouassi, T. D. et Yalinkpon, F. 2019. Caractérisation agro-morphologique des meilleurs clones d'anacardiers (Anacardium occidentale L.) du Bénin. Bulletin de la Recherche Agronomique du Bénin (BRAB), Numéro spécial (2019) : 41-53. ISSN 1025-2355.

Bezerra, M.A., De Lacerda, C.F., Filho, E.G., De Abreu, C.E.B. et Prisco, J.T. 2007. Physiology of cashew plants grown under adverse conditions. Brazilian Journal of Plant Physiology 19(4):449-461.

Bognina, A., Guira, M., Yameogo, J.T., Tarpaga, V. et Rouamba, A. 2019. Essai de multiplication par greffage d'une accession d'anacardier à grosses pommes à la Station de recherche de Banfora au Burkina Faso. Afrique Science 15(4):156-168.

Das, P., Sreelatha, T. et Ganesh, A. 2004. Biooil from pyrolysis of cashew nut shellcharacterization and related properties. Biomass and Bioenergy 27:265-275.

Dasmohapatra, R., Rath, S., Pradhan, B. et Rout, G.R. 2014. Molecular and agromorphological assessment of cashew (Anacardium occidentale L.) genotypes of India. Journal of Applied Horticulture 16 (3):215-221.

De Figueiredo, R.W, Lajolo, F.M, Alves, RE. et Filgueiras, H.A.C. 2001. Physicalchemical changes in early dwarf cashew pseudofruits during development and maturation. Food Chemistry 77:343-347.

De Laroussilhe, F. 1980. Le Manguier. Techniques Agricoles et Productions Tropicales. Éditions G-P. Maisonneuve et Larose: Paris. 312pp.

Djaha, J.B., N'Daadopo, A.A., Koffi, E.K., Ballo, C.K. et Coulibaly, M. 2012. Croissance et aptitude au greffage de deux génotypes d'anacardier (Anacardium occidentale L.) élites utilisés comme portegreffe en Côte d'Ivoire. International Journal of Biological and Chemical Sciences 6(4) 1453-1466. doi : http:// dx.doi.org/ 10.4314/ijbcs.v6i4.5

Djaha, A.J-B., N'da, H.A., Koffi, K.E., N'da Adopo, A. et Ake, S. 2014. Diversité morphologique des accessions d'anacardier (Anacardium occidentale L.) Introduits en côte d'ivoire. Revue Ivoirienne des Sciences et Technologie 23:244-258.

Dwomoh, E.A., Ackonor, J.B. et Afun, J.V.K. 2008. Survey of insect species associated with cashew (Anacardium occidentale Linn.) and their distribution in Ghana. African Journal of Agricultural Research 3:205-214.

Eteka, A.C. et Faaki, V.A. 2017. Etude diagnostique sur les contraintes et les opportunités à l'accès aux intrants dans les maillons de production et de transformation au Bénin. DEDRAS-ONG Organisation 
pour le Développement Durable, le Renforcement et l'Autopromotion des Structures communautaires, Parakou, Bénin. 55pp.

Food and Agricultural Organization (FAO). 2009. Statistiques rapportées par www.cashewinfo.com.

Food and Agricultural Organization (FAO). 2014. Base des données de la FAO 2011. http://faostat3.fao.org.

Food and Agricultural Organization (FAO). 2020. Production des cultures en Afrique. Données 2018. http://faostat.fao.org/site, mise à jour le 4 mars 2020, révision mineure.

Hammed, L.A., Amnikwe, J.C. et Adededji, A.R. 2008. Cashew nuts and production development in Nigeria. American-Eurasian Journal of Scientific Research 3(1):54-61.

Hala, N., Dembélé, B., N'da Adopo, A.A., Coulibaly, F., Kehe, M., N'goran, Y.A. et Doumbia, M. 2011. Population dynamics of the mango mealybug, Rastrococcus invadens William (Homoptera: Pseudococcidae) in northern Côte d'Ivoire. Journal of Animal and Plant Sciences 12(1):1481-1492. doi: https://www.ajol. info/index.php/aga/artic le/viewFile/ $100648 / 89863$.

Lacroix, E. 2003. Les anacardiers, les noix de cajou et la filière anacarde à Bassila et au Bénin. Projet de Restauration des Ressources Forestières de Bassila, GFA Terra Systèmes and GIZ. 75pp.

Lautié, E., Dorniera, M., De Souza, F.M. et Reynes, M. 2001. Les produits de l'anacardier : caractéristiques, voies de valorisation et marchés. Cirad. Fruits 56(4):235-248.

Lawal, J.O., Oduwole, O.O., Shittu, T.R. et Muyiwa, A.A. 2010. Profitability of value addition to cashew farming households in Nigeria. African Crop Science Journal 19(1):49-54.

Martin, P.J., Topper, C.P., Bashiru, R.A., Boma, F., De Waal, D., Harries, H.C., Kasuga, L.J., Katanila N, Kikoka L.P.,
Lamboll R., Maddison A.C., Majule A.E., Masawe P.A., Millanzi, K.J., Nathaniels, N.Q., Shomari, S.H., Sijaona, M.E. et Stathers, T. 1997. Cashew nut production in Tanzania: Constraints and progress through integrated crop management. Crop Protection 16(1):5-14.

Martinez, A.R., Penarredona, M.A., Pheng, B., Hoyos, D.E., Ting, J.C.H. et Alvarez, N.F.P. 2011. Global Enterprise Experience, INDICASHEW, TEAM 58. 8pp.

Monteuuis, O. 1988. Aspects du clonage de sequoias géants jeunes et âgés. Thèse de Doctorat de l'Université Clermont-Ferrand II, France. 190pp.

N'Djolossè, K., Kodjo, S., Dah-Dovonon, Z.J., Badou, A., Maliki, R., Tandjiékpon, M.A., Salifou, M. et Agbo, P.B. 2015. Sélection d'arbres-mères d'anacardiers performants pour la production de plants greffés au Bénin. Poster. Dépôt légal $N^{\circ} 8318$ du 16/12/2015 Bibliothèque Nationale du Bénin, ISBN: 978-99919-0900-4.

N'Djolossè, K., Adoukonou-Sagbadja, H., Gbèmavo, C.D. S. J., Kodjo, S., Badou, A., Maliki, R. et Adjovi, R.N.A. 2019. Agro-morphological characterization of preselected cashew (Anacardium occidentale L.) mother trees in Benin farmer's plantations. Journal of Agriculture and Environment for International Development 113(1):17-34.

PAC/DCM/SESP. 2009. Evolution du trafic marchandises: Période 1999 à 2008.

Santos, R.P., Santiago, A.A.X., Gadelha, C.A.A., Cajazeiras, J.B., Cavada, B.S., Martins, J.L., Oliveira, T.M., Bezerra, G.A. et Freire, V.N. 2007. Production and characterization of the cashew (Anacardium occidentale L.) peduncle bagasse ashes. Journal of Food Engineering 79:1432-1437.

Sedia, N.A.G. 2001. Introduction à la culture de l'anacardier et renégociation des règles des jeux fonciers et sociaux : l'exemple de Baradougou et de Touro en zone de contact 
forêt-savane. Mémoire de D.E.A, Université de Bouaké, Côte d'Ivoire. 68pp.

Soloviev, P., Niang, T.D. et Gaye, A. 2004. Propagation par greffage du prunier d'Afrique (Sclerocarya birrea (A. Rich.) Hochst.) au Sénégal. Fruit 59(4):275-280. DOI : https://www.ajol.info/index. php/ajb/ article/download/.../82452.

Tandjiékpon, A.M., Lagbadohossou, A., Hinvi, J. et Afonnon, E. 2003. La culture de l'anacardier au Bénin : Référentiel Technique. Edition INRAB, ISBN 9991951-66-0. 86pp.

Tandjiékpon, A.M. 2010. Analyse de la chaine de valeur du secteur anacarde du Bénin. Initiative du Cajou Africain (ICA), Cotonou. 64pp.

Thimmappaiah, W.G., Santhosh, D.S. et Melwyn, G.S. 2009. Assessment of genetic diversity in cashew germplasm using RAPD and ISSR markers. Scientia Horticulturae 120:411-417.

Toure, M.A., Faye, E. et Gondiaby, R. 2017. Réponse de quatre variétés de Anacardium occidentale L. aux techniques de greffage horticole en pépinière, - Revue électronique en sciences de l'environnement VertigO.

Trepko, P. 2003. La culture de l'anacardier dans la Région de Bassila au Nord Bénin. Projet de restauration des ressources forestières de Bassila, République du Bénin, GTZ. 53pp.

Yabi, I., Yabi Biaou, F. et Dadegnon, S. 2013. Diversité des espèces végétales au sein des agro-forêts à base d'anacardier dans la commune de Savalou au Benin. International Journal of Biological and Chemical Sciences 7(2):696-706. 